\title{
Decoding Personality Trait Measures from Resting EEG: An Exploratory Report
}

\author{
Hayley K. Jach, Daniel Feuerriegel, and Luke D. Smillie \\ Melbourne School of Psychological Sciences, The University of Melbourne
}

Can personality be predicted from oscillatory patterns produced by the brain at rest? To date, relatively few electroencephalographic (EEG) studies have yielded consistent relations between personality trait measures and spectral power, suggesting that new exploratory research may help develop targeted hypotheses about how neural processes associated with EEG activity may relate to personality differences. We used multivariate pattern analysis to decode personality scores (i.e., Big Five traits) from resting EEG frequency power spectra. Up to 8 minutes of EEG data was recorded per participant

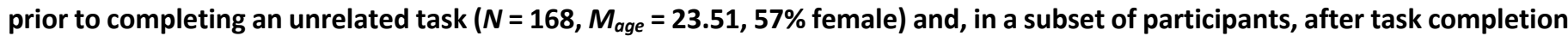
( $N=96, M_{\text {age }}=23.22,52 \%$ female), measuring the resting state with open and closed eyes. Linear support vector regression with 10-fold cross validation was performed using the power from 62 scalp electrodes within $1 \mathrm{~Hz}$ frequency bins from 1-30 $\mathrm{Hz}$. One Big Five trait, agreeableness, could be decoded from EEG power ranging from 8-19 $\mathrm{Hz}$, and this was consistent across all four recording periods. Neuroticism was decodable using data within the 3-6 Hz range, albeit less consistently. Posterior alpha power negatively correlated with agreeableness, whereas parietal beta power positively correlated with agreeableness. We suggest methods to draw from our results and develop targeted future hypotheses, such as linking to individual alpha frequency and incorporating self-reported emotional states. Our open dataset can be harnessed to reproduce results or investigate new research questions concerning the biological basis of personality.

Keywords Multivariate pattern analysis | power spectra | support vector regression | exploratory | personality traits

This manuscript has been accepted for publication at Cortex. This version has not been copy-edited. Open data and analysis code can be found at the following link: https://osf.io/3mnt7/

$\mathbf{P}_{\mathrm{e}}$ ersonality traits describe enduring patterns of emotions, thoughts, and behavior. Personality traits are heritable (Bleidorn, Kandler, \& Caspi, 2014), relatively stable across the lifespan (Specht, Egloff, \& Schmukle, 2011; Bleidorn, Hopwood, \& Lucas, 2018), and predict important life outcomes such as romantic satisfaction, occupational success, financial security, substance abuse, and criminal behavior (Ozer \& Benet-Martinez, 2006; Soto, 2019). Researchers have dedicated almost a century to describing and refining the structure of personality traits (Allport, 1937; Digman, 1990), resulting in the dominant Big 5 model of personality (John, Naumann, \& Soto, 2008). The Big 5 model decomposes trait variance into five core dimensions: agreeableness (pro-sociality, compassion, and politeness), neuroticism (anxiety, depression, and emotional volatility), conscientiousness (organization and action regulation in goal-directed behavior), extraversion (sociability, enthusiasm, and assertiveness), and openness/intellect (imagination, curiosity, and aesthetic sensitivity).

The growing literature of personality neuroscience investigates how personality traits relate to various neurobiological indices, with an ultimate goal to understand the mechanistic underpinnings of personality (DeYoung \& Gray, 2009; Allen \& DeYoung, 2017). In this area, researchers have correlated measures of neural activity-using methods such as electroencephalography
(EEG) and functional magnetic resonance imaging (fMRI)with personality trait measures. These measurements are often taken when participants are at rest and are not engaged in a predefined task. As personality traits are theorized to reflect relatively stable and sustained cognitive and emotive processes, we might expect patterns of neural activity reflecting or facilitating these processes to be detectable in such resting-state recordings. For example, individuals scoring higher in openness/intellect are more likely to imagine and daydream, as measured by retrospective self-report and experience sampling (Kane et al., 2017; Zhiyan \& Singer, 1997), and increased daydreaming seems likely to occur at rest. There have, in turn, been several studies observing that openness/intellect relates to increased connectivity within the default network (Beaty et al., 2016, 2018), a collection of cortical regions implicated in a variety of self-reflective processes, including imagination. Relations between personality traits and resting-state activity have been found for each of the Big 5 personality traits (see Allen \& DeYoung, 2017 for a review).

The majority of studies investigating personality correlates of resting-state activity have used fMRI connectivity metrics, which are valuable for identifying covariation in activity across brain regions that are active over the resting period. An alternative and complementary approach is to measure the brain's rhythmic oscillatory 
patterns: that is, dynamic patterns of energy in given frequency bands (alpha, beta, theta, etc.; Buzsáki, 2006). For this task, EEG-which has much higher temporal precision than $\mathrm{fMRI}$ - is the method of choice. Researchers have extensively documented typical oscillatory behaviors related to behavior and cognition at rest and in response to task demands (for a review, see Knyazev, 2007) and there is a growing interest in exploring individual differences in oscillatory activity, providing insights into how sensory stimulation is integrated into percepts over time (Cecere, Rees, \& Romei, 2015), and differences in cognitive abilities (e.g., Grandy et al., 2013; Klimesch et al., 1996) or clinical populations (e.g., Schizophrenia and Attention Deficit Disorder, Başar \& Güntekin, 2013; Autism Spectrum Disorders, Cornew, Roberts, Blaskey, \& Edgar, 2012).

Investigations of the personality correlates of resting-state EEG activity have so far failed to yield robust and replicable findings. The largest collection of studies has concerned frontal alpha asymmetry (i.e., alpha power over right relative to left frontal cortical areas), which is thought to index processes associated with extraversion and neuroticism (Harmon-Jones, 2003; Wacker \& Smillie, 2015). However, in a recent meta-analytic study combining all published studies (and a number of unpublished studies), Kuper, Käckenmester, and Wacker (2019) reported negligible effects, with less than $0.4 \%$ of the variance in extraversion and neuroticism explained by frontal alpha asymmetry. This is concerning for researchers working to integrate empirical results into overarching mechanistic theories in personality neuroscience (e.g., Kennis, Rademaker, \& Geuze, 2013). If the evidence base drawn upon to develop theories in personality neuroscience is not robust, then the resultant theories will not be useful.

These knowledge gaps within the resting-state EEG literature present a challenge to personality neuroscience, but also signal an opportunity for new research to thoroughly explore how personality traits relate to EEG indices. One powerful tool for data-driven research is Multivariate Pattern Analysis (MVPA). Many techniques that fall under the umbrella of MVPA consider the joint contributions of multiple features of data simultaneously, whether they be multiple voxels when using fMRI or signals from multiple electrodes in the EEG (Grootswagers, Wardle, \& Carlson, 2017). A key advantage of MVPA is that it can efficiently explore a large feature space to identify signals that can predict the outcome of interest in a data-driven fashion. MVPA has been extensively applied to fMRI data (see Tong \& Pratte, 2012, for a review), and its usage in EEG has grown over the past decade. To date, researchers have used MVPA to decode a range of neural correlates of perceptual and cognitive processes, including sensory representations (Hogendoorn, Verstraten, \& Cavanagh, 2015), face detection (Cauchoix, Barragan-Jason, Serre, \& Barbeau, 2014), perceptual decisions (Bode et al., 2012), and evaluations of the subjective relevance of everyday objects (Turner, Johnston, de Boer, Morawetz, \& Bode, 2017).

To our knowledge, only one study (Korjus et al., 2015) has used MVPA-specifically support vector machine classification-to assess whether personality can be predicted from resting EEG frequency power data. They used a median split to categorize those low or high on a given trait and attempted to discriminate between low/high personality trait groups using frequency band power estimates across 32 scalp channels. Although the models used could classify extraversion and openness/intellect at above chance levels, this was not statistically significant after corrections for multiple comparisons. Despite the strengths of this study, including a large sample $(N=289)$ and sophisticated analyses, there are several reasons why further research of this kind is required in order to be confident in its somewhat pessimistic conclusions. First, most participants provided only short periods of resting data (1 or 2 minutes, compared to 8-10 minutes in other studies; Wacker, Chavanon, \& Stemmler, 2010), which may yield a relatively low signal to noise ratio when obtaining frequency domain power estimates. The authors also did not report the internal consistency of their personality measures, making it unclear how reliable the personality scales were. Poor reliability indicates that the same participants are responding inconsistently to survey items within a single Big 5 trait scale, suggesting that the scale did not successfully tap a latent construct (Cronbach, 1951). Trying to predict something that was not measured precisely would clearly reduce model performance. Additionally, transforming Likert-scale responses to binary high-low categories is well-known to lower statistical power and potentially produce misleading results (MacCallum, Zhang, Preacher, \& Rucker, 2002). As there were approximately Gaussian distributions of personality trait scores across their sample, this also meant that many participants on each side of the median split would have provided very similar personality trait scores. In addition, the authors used binomial tests to determine statistical significance, which requires a threshold level of classifier performance (here a $\sim 57 \%$ correct classification rate) to reach statistical significance. If the factors mentioned above did hinder classifier performance, then even in the presence of real patterns that distinguish participants with low and high trait scores, the classifiers may not have met these cutoffs. Korjus and colleagues (2015) did conduct additional analyses using continuous trait measures using LASSO regression; however, they explored a very large feature space for optimizing the model in their analyses, including selection of task conditions (eyes open/closed), frequencies, electrode sets, and model fitting parameters. With such a large feature space relative to the number of observations, it is possible that their sparse regression approach may not have selected the most informative features in their data.

Given the dearth of research in this area, we attempted to predict Big 5 personality trait scores from resting state EEG data using a multivariate analysis technique well-suited to continuous outcome variables. As our study was entirely exploratory, we did not propose any hypotheses. However, our analyses were designed to provide comprehensive results that minimized inferences from noise. Full details of our approach are detailed in the Method, but we highlight key contributions here. First, we assessed the performance of models trained using power in particular frequency bands in separate analyses, rather than assessing all of the EEG data at once. This allowed us to ascertain which spectral bands may be most relevant to personality trait measures. Second, we assessed 
consistency across multiple testing conditions by collecting resting data at two time points: before and after completing a task that was unrelated to the current study. At each of these time points, we further assessed consistency across alternating periods of eyes-open versus eyes-closed recordings. This would account for effects that may be linked to the specifics of the testing session (e.g., the effect of having one's eyes open or closed; the effect of collecting data before or after the unrelated task). And third, we decided a priori to only make inferences from results where decoding performance was close to $r=.20$ in all four conditions, at approximately the same frequency bands for each condition, according to the following rationale. First, $r$ $=.20$ is the average effect size in personality psychology (Giznac \& Szorroli, 2016), and given that we are conducting a purely exploratory study, it seemed appropriate to anchor our predictions to the average effect size in the field. Second, $r=.20$ is the effect size that we have approximately $80 \%$ power to detect given our sample size using traditional methods, suggesting it would be unwise to trust inferences less than $r=.20$. And third, we would expect any true correlation between a neurobiological measure and a personality scale to be relatively small (Pickering \& Pesola, 2014). Specifically, there are many possible causes for responses to any item on a personality scale, and there are also many possible causes for the recorded EEG signal. If there is a true relationship between oscillatory patterns and personality, then this suggests that some of the causes from the EEG signal overlap with some of the causes from the items that make up a personality measure. However, because the shared causes could only represent a small subset of the causes that contribute shared variance to a personality trait, any observable correlation between a personality trait and a neurobiological measure would not be expected to be higher than, at an optimistic estimate, the average effect size in personality research. Thus, assessing the traits that are consistently predicted across conditions at $r=.20$ is a further guard against over-interpreting spurious findings.

\section{Method}

\subsection{Open Science Practices}

Our materials, data, and analysis code are available so that others may view the data, reproduce our analyses, and conduct additional analyses (accessible from https://osf.io/3mnt7).

\subsection{Participants and Experimental Procedure}

Data were provided by 210 participants $\left(M_{\text {age }}=\right.$ $23.85, S D_{\text {age }}=5.48,59 \%$ female) who took part in a variety of EEG experiments in our lab (e.g., Hughes et al., 2015; Smillie et al., 2019) and provided personality trait ratings on a 5-point Likert scale via the Big 5 Aspects Scale (BFAS; DeYoung, Quilty, \& Peterson, 2007). As we used existing data, sample size was determined by the number of participants that had previously been collected.
There was no manipulation in this study. Before completing tasks unrelated to the current study, participants were asked to sit at rest, alternating between open and closed eyes every 60 seconds when prompted by the experimenter. This resulted in approximately eight minutes of data per participant, comprising four minutes eyes closed, and four minutes eyes open. We collected pretask resting data for 205 participants $^{1}$. For a subset of 102 participants, post-task resting data was also obtained approximately 60 minutes after the first resting state recording, in the same manner as the pre-task data.

Following EEG data pre-processing (Section 2.3) we retained 168 participants in the pre-task conditions $\left(M_{\text {age }}=\right.$ $23.51, S D_{\text {age }}=5.32$, range $=18-41,57 \%$ female), hereafter labeled pre-task eyes open and pre-task eyes closed; and retained 96 participants in the post-task conditions $\left(M_{\text {age }}=\right.$ $23.22, S D_{\text {age }}=4.77$, range $=18-37,52 \%$ female), labeled post-task eyes open and post-task eyes closed. Note that 78 individuals provided only pre-task data, 6 individuals provided only post-task data, and 90 participants provided both pre-and post-task data. This brings the total number of personality survey responses to $N=174$, and this is the sample that is used for internal consistency estimates and descriptive statistics (calculated using the Psych package, as implemented in R; Revelle, 2018).

\subsection{EEG Acquisition and Pre-Processing}

We recorded EEG from 64 active scalp electrodes using a Biosemi Active Two system (Biosemi, the Netherlands). Recordings were grounded using common mode sense and driven right leg electrodes (http://www.biosemi.com/faq/cms\&drl.htm). To this montage we added two electrodes located $1 \mathrm{~cm}$ from the outer canthi of each eye, and two electrodes above and below the right eye. EEG was sampled at $512 \mathrm{~Hz}$ (DCcoupled with an anti-aliasing filter, $-3 \mathrm{~dB}$ at $102 \mathrm{~Hz}$ ). We attempted to keep electrode offsets within $\pm 40 \mu \mathrm{V}$, although this wasn't always possible (see below for description of removed channels).

All pre-processing was conducted in Brain Vision Analyzer Version 2.1.2 (Brain Products $\mathrm{GmbH}$, 2017). To aid reproducibility, the full EEG processing pipeline used for this analysis can be found in our data documentation. EEG data were filtered with IIR zero-phase Butterworth filters $0.5 \mathrm{hz}$ high-pass with time constant 0.318 and order 2; 30hz low-pass with order 2; 50hz notch) and an initial manual check for flat-lined or artefact-ridden channels was conducted; if found, these were excluded from subsequent processing steps (re-referencing, manual artefact removal and ocular artefact removal), and later interpolated (i.e., estimated from surrounding channels) as noted below. We made an a priori decision to remove a dataset if more than four channels appeared to be artefact-ridden or flat-lined. This resulted in the removal of 37 datasets in the pre-task condition, and 6 datasets in the post-task condition ${ }^{2}$. Our full data processing records are included with the open data and code.

\footnotetext{
${ }^{1}$ Five participants provided survey data but did not take part in the EEG experiment.

${ }^{2}$ Much of the data was originally collected to assess frontal activity (e.g.,

Hughes et al., 2015), and faulty or poorly connected channels in the
}

parietal/occipital areas were frequently tolerated to expedite data collection. As a result, many more participants were removed here than might be expected. 
We initially referenced the data to channel $\mathrm{Cz}$ (or, for 12 datasets where $\mathrm{Cz}$ appeared to be dead/artefact ridden, to $\mathrm{CPz}$ ). Sections of data with muscle artefacts or skin potentials were identified by visual inspection and removed. Data were segmented into 1-minute epochs timelocked to the onset of "eyes open" and "eyes closed" cues, and an Independent Components Analysis (ICA) was performed using an infomax (gradient) restricted algorithm, classic PCA sphering (such that all components are treated equally), a convergence bound of $1 \mathrm{E}-07$, and a maximum of 512 iterations. Components corresponding to eye blinks and saccades were removed. Following ICA, we interpolated any excluded channels using spherical splines ( $4^{\text {th }}$ order) and subsequently re-referenced to the average of all scalp channels. Next, data were segmented into 2second epochs with a 1-second overlap. Segments were tapered using a Hamming window. Segments $200 \mathrm{~ms}$ before and after artefacts were excluded from analyses using an automated rejection algorithm (where artefacts were considered to be any of the following: greater than $50 \mathrm{uV}$ difference in voltage per millisecond; greater than $200 \mathrm{uV}$ difference in a $200 \mathrm{~ms}$ interval; less than $0.5 \mathrm{uV}$ difference between the maximum and minimum voltage in a $100 \mathrm{~ms}$ interval). Segments of EEG data were converted into frequency domain representations using fast Fourier transforms $(0.5 \mathrm{~Hz}$ frequency resolution), and the average amplitudes in each frequency bin across segments were computed for each eyes open and eyes closed condition. Power in frequency bins ranging from $0.5-30 \mathrm{~Hz}$ were used for MVPA.

\subsection{Multivariate Pattern Analyses (MVPA) of EEG Power Spectra}

The Decision Decoding Toolbox (DDTBox; Bode, Feuerriegel, Bennett, \& Alday, 2018) was used to perform support vector regression (SVR). Plots of decoding performance (Figure 1 and 2) were computed in R version 3.6.1 (R Core Team, 2018) using ggplot2 (Wickham, 2016).

The feature space used across different analyses consisted of frequency spectra including 30 frequency bins ranging from $0.5-30 \mathrm{~Hz}$ at 62 scalp channels ${ }^{3}$. Sets of $0.5 \mathrm{~Hz}$ frequency bins were averaged to create the 30 frequency bins used for MVPA. For example, the $1 \mathrm{~Hz}$ frequency bin used for MVPA consisted of data from the $0.5 \mathrm{~Hz}$ and $1 \mathrm{~Hz}$ frequency bins produced by the FFTs.

Participants' scores for a given personality trait (e.g., neuroticism) were used as the values to be predicted in the regression models. We performed separate regression analyses for each condition (pre eyes open, pre eyes closed, post eyes open, post eyes closed), and each personality trait (neuroticism, openness/intellect, extraversion, agreeableness, and conscientiousness), resulting in 20 separate conditions. For each condition, we used SVR with a linear kernel, as implemented in LIBSVM (Chang \& Lin, 2011), to predict personality trait scores from the joint patterns of spectral power over all 62 channels within a given frequency bin (e.g., 1Hz). The analysis was then repeated for each of the remaining frequency bins from 2 to $30 \mathrm{~Hz}$.

\footnotetext{
${ }^{3}$ We analyzed the data without $\mathrm{Cz}$ and $\mathrm{CPz}$, as these had been used as reference electrodes during data processing. As a robustness check, we assessed decoding performance both with and without these electrodes
}

With this number of individual regressions and conditions-and given that our sample size is smaller than the number at which correlations of around $r=.20$ typically stabilize ( $n \sim 250$; Schönbrodt \& Perugini, 2013)-it is possible that some frequency bins may show high decoding performance by chance. However, we implemented several steps to guard against making inferences from potentially spurious high values. First, to reduce the influence of random variation in decoding performance, we used 10fold cross-validation procedures for all analyses. Crossvalidation is the recommended practice to avoid data overfitting and to increase the stability of estimates (Arlot \& Celisse, 2010). For each SVR analysis, the dataset was split into ten subsets; the model was trained on nine of the subsets and tested on the remaining subset. This process was repeated nine times with a different subset allocated as the test data each time. To minimise any drawing biases from the particular subset of data, we repeated this entire cross-validation procedure 10 times ( 5 times for permuted labels). In other words, we obtained the average of 100 correlation coefficients per frequency bin, which should reduce the impact of outliers in decoding performance. Following this procedure, we averaged decoding performance measures over analysis repetitions (following Turner et al., 2017).

To provide a further safeguard against overinterpretation, we assessed consistency in model performance for each personality trait in four conditions (pre eyes open, pre eyes closed, post eyes open, post eyes closed) via the Fisher-transformed correlation coefficients between predicted and actual labels. As previously mentioned in Section 1, we decided a priori to only make inferences from results where decoding performance was close to $r=.20$ in all four conditions, at approximately the same frequency bands for each condition.

Finally, in addition to our SVR analyses predicting personality trait scores, we also randomized which personality scores were assigned to each participant dataset and performed SVR on this resulting EEG data with permuted labels. Permuted-labels decoding allowed us to empirically estimate the chance level of decoding performance, which is often a more stringent control than comparisons against theoretical chance level (Combrisson \& Jerbi, 2015). We then compared decoding performance in the permuted labels analyses with that of the original analyses across different frequency bands. Although it is possible to compute a $p$-value by comparing actual decoding performance to a null distribution of e.g., 1,000 iterations of permuted labels decoding, we did not calculate $p$-values here so as not to incorrectly imply that our analyses were confirmatory. This aligns with recommendations for exploratory studies to focus on estimation of effect sizes and their uncertainty, rather than on significance testing (Szucs \& Ioannidis, 2017).

\subsection{Post-hoc Spectral Power Correlations}

Although above-chance decoding can indicate that information related to personality traits is present in the resting EEG data, this does not inform us as to the direction of such relations between neural and personality measures.

included, and results were highly similar. The linked data repository includes these electrodes such that researchers are able to replicate results with the full 64 channels. 
Thus, for any above-chance decoding for a given personality trait that was consistent across the four conditions in any frequency band, we conducted post-hoc Spearman correlations (de Winter, Gosling, \& Potter, 2016) between scores for that trait and measures of power within each frequency bin at each channel. This permitted assessment of any clear monotonic relations between spectral power and personality trait scores. Given the number of correlations computed for this analysis, there is likely to be some imprecision around the estimates. However, again, we focus on results that are highly similar across the four conditions (pre-task eyes open, pre-task eyes closed, post-task eyes open, post-task eyes closed).

\section{Results}

\subsection{Descriptive Statistics for Personality Traits and EEG Measures}

Means, standard deviations, reliability (omega total), and correlations between personality traits are presented in Table 1. All traits displayed strong internal consistency. Medium-to-large correlations could be observed between several personality traits: for example, agreeableness, extraversion, and openness/intellect. These patterns of intercorrelations are commonly observed in the literature (e.g., DeYoung, 2006) and support the assumption that out data are representative of typical personality survey scores. Supplementary Figure S1 compares the distributions of trait scores in the pre- $(N=$ $168)$ and post- $(N=96)$ conditions, including correlations between traits in each of these conditions. These were very similar, suggesting that any differences in model decoding across pre- and post- conditions would not be due to differences in personality trait distributions.

Supplementary Figure S2 displays spectral power patterns at different frequency bands averaged across participants. EEG data were representative of what is typically observed in the literature (Buzsáki, 2006) suggesting that decoding results may also be representative of what is found in the wider population of individuals.

\subsection{MVPA of Frequency Band Power Estimates}

Figure 1 displays the mean decoding performance over cross-validation folds, analysis repetitions, and pre/post eyes-open/eyes-closed conditions for each of the Big 5 traits, with Panel F providing a summary of the results for all traits. The supplementary information includes exact correlations obtained at each frequency bin.

For two of the five traits (agreeableness and neuroticism), scores could be predicted from the EEG data across multiple conditions. Of these, agreeableness (Figure 1 , panel A) emerged as the trait that was most consistently predicted across the largest number of frequencies. The correlation between model-predicted agreeableness scores and survey scores was around $r=.20$ between approximately 8 and $19 \mathrm{~Hz}$. Assessing agreeableness results from each eyes open/closed and pre/post task conditions (Figure 2, Panel A), there was higher variability in decoding performance when compared to the conditionaveraged plots. Despite this, it is striking how consistently the model predicts agreeableness, particularly in the range of $12-18 \mathrm{~Hz}$ (the lower beta band).

Neuroticism also appeared to be consistently predicted by the model at levels above chance, in this instance between approximately 3 to $6 \mathrm{~Hz}$ (i.e., the theta band). For pre-task eyes open and pre-task eyes closed conditions (Figure 2B, first and second plot), correlations hovered around $r=.20$ in this frequency band. For the two post-task conditions (Figure 2B, third and fourth plot), the same pattern emerged, but the correlations were smaller (post-task eyes open $r \sim .10$; post-task eyes closed $r \sim$.16). Between 10-12 Hz there were somewhat consistent negative correlations between the model-predicted and actual neuroticism scores across conditions, which can also be seen in the condition-averaged plots for extraversion, openness/intellect, and conscientiousness. It is difficult to interpret decoding performance that is worse than chance level; however, this may hint at subgroups of participants that show different relations between neuroticism scores and EEG activity, or nonlinear relationships between EEG power and personality trait scores. Models trained predominantly on one subset, and tested on those from a different subset, could produce below-chance performance as seen here. For example, co-occurring differences in the individual alpha peak frequency and alpha band amplitude (e.g., Klimesch, Doppelmayr, Schwaiger, Auinger, \& Winkler, 1999) might produce such nonlinear relationships.

Table 1.

Means, Standard Deviations, Internal Consistency, and Correlations Between Personality Traits

\begin{tabular}{lccccccc}
\hline \multicolumn{1}{c}{ Variable } & $M$ & $S D$ & $\omega$ & 1 & 2 & 3 \\
\hline 1. Agreeableness & 3.91 & 0.45 & .87 & & & & \\
2. Conscientiousness & 3.35 & 0.52 & .87 & .11 & & \\
3. Extraversion & 3.42 & 0.54 & .90 & .32 &. $\mathbf{3 0}$ & \\
4. Neuroticism & 2.86 & 0.65 & .92 & -.08 & -.35 & -.26 &. $\mathbf{2 6}$ \\
5. Openness/Intellect & 3.77 & 0.46 & .78 &. $\mathbf{2 5}$ & .15 & -.05 \\
\hline
\end{tabular}

Note. $M$ and $S D$ represent mean and standard deviation, respectively. $\omega=$ omega total (internal consistency; roughly equivalent but superior to Cronbach's alpha). Italic font indicates $p<.05$. Bold font indicates $p<.01$. 
A Agreeableness

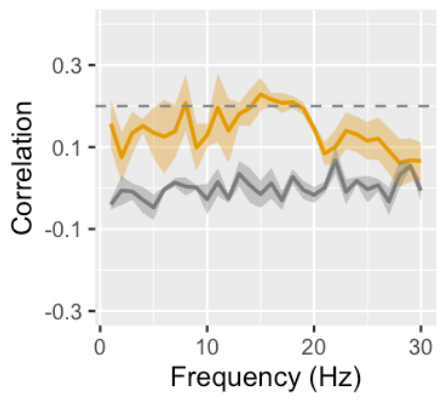

D Openness/Intellect

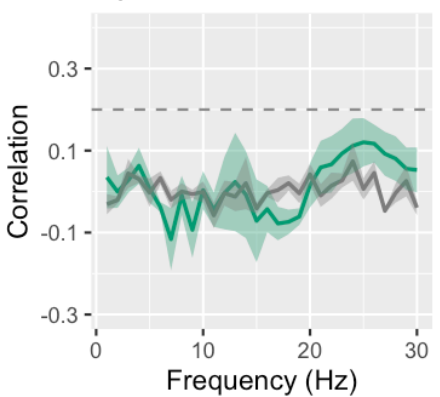

B Neuroticism

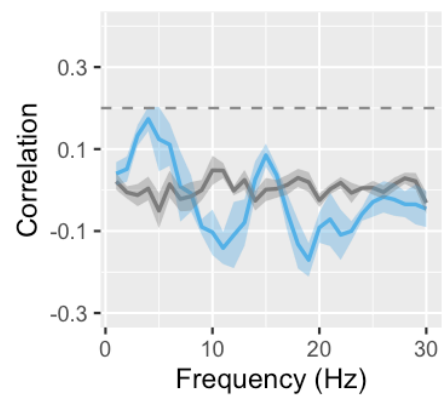

E Extraversion
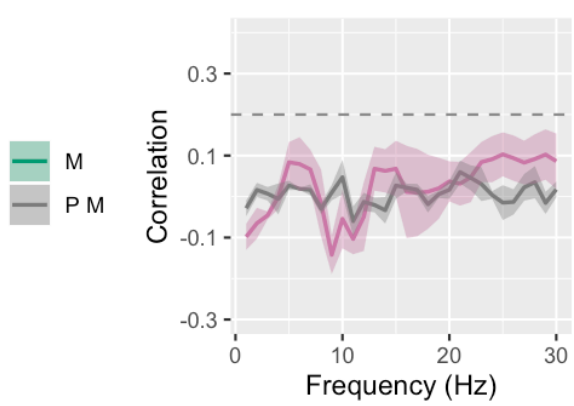

C Conscientiousness

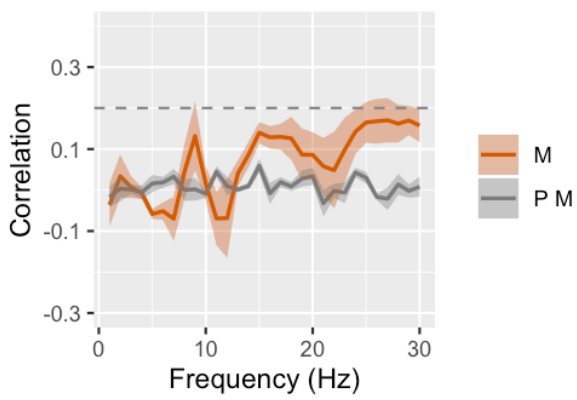

F All Traits

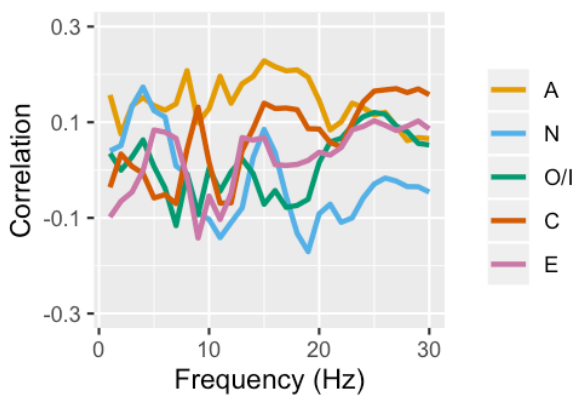

Figure 1. Mean decoding performance for each personality trait (A through E) and all traits combined (F). Colored lines depict decoding performance for real personality survey responses, and gray lines depict decoding performance of models trained using permuted labels. A dotted horizontal line at $r=.20$ is included to compare decoding performance to this threshold. Agreeableness is consistently decoded at approximately $r=.20$ across 8 to 19 Hz frequency bins, whereas neuroticism is consistently decoded close to $r=.20$ from 3-6hz. For A-E, error bars depict the standard error of correlation coefficients across the four conditions (pre eyes-open, pre eyes-closed, post eyes-open, and post eyes-closed). $\mathrm{M}=\mathrm{Mean}$ Correlation Coefficient, PM = Permuted-Labels Analysis Mean Correlation Coefficient.

A Agreeableness Pre EO

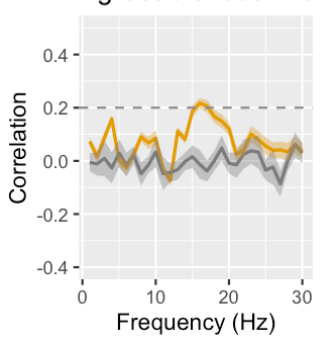

B

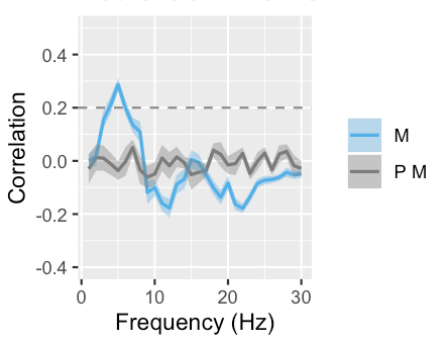

Agreeableness Pre EC

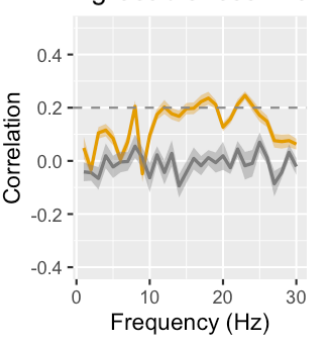

Neuroticism Pre EC

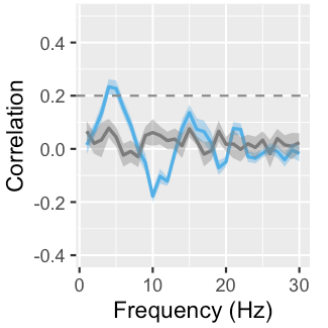

Agreeableness Post EO

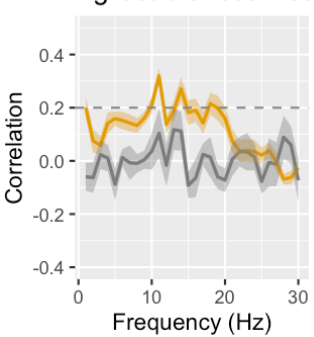

Neuroticism Post EO

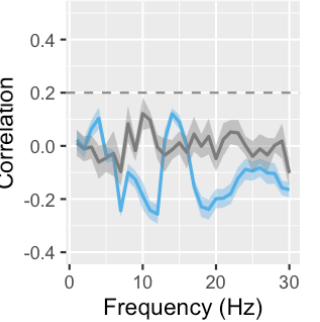

Agreeableness Post EC

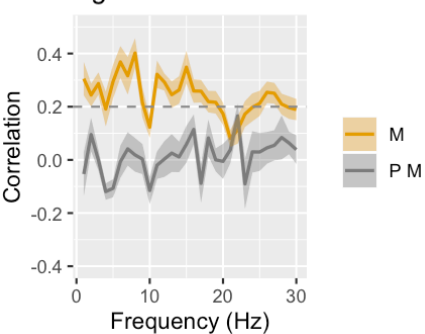

Neuroticism Post EC

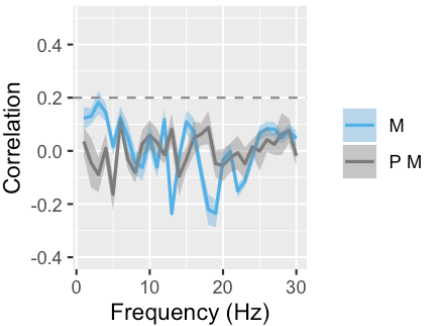

Figure 2. (A) Decoding performance for each condition of agreeableness, all four conditions. (B) Decoding performance for neuroticism, all four conditions. Error bars depict the standard error of the 10 repetitions of 10 -fold cross-validation. $\mathrm{EO}=$ Eyes open, $\mathrm{EC}=$ Eyes closed, $\mathrm{M}=\mathrm{Mean}$ Correlation Coefficient, PM = Permuted-Labels Analysis Mean Correlation Coefficient. 
Conscientiousness also appeared to be predicted by the model, specifically in the upper beta band (2330hz). However, the mean result shown in Figure 1 appears to have been driven by very high decoding performance in one condition: post-task eyes closed (see Figure S3, panel C). Here, correlations around $r=.30$ were observed for all of these frequency bins. Other conditions did show some evidence of decodable information, but effect sizes were very small: the post- and pre-task eyes closed conditions were around $r=.14$, and the pre eyes open condition produced a correlation between surveypersonality and model-predicted personality of $r=.08$. We suggest that given this variability, results are not as convincing as those for the other two traits, particularly agreeableness.

Scores for openness/intellect and extraversion could not be reliably decoded across conditions (Supplementary Figures S4 and S5).

\subsection{Post-Hoc Correlation Analyses}

As agreeableness and neuroticism were consistently decoded at above-chance levels across preand post-task conditions, we assessed the patterns of correlations between personality trait scores and power at each channel and frequency bin. Figure 3 displays Spearman correlation coefficients describing strength of association between spectral power and personality trait scores across all 62 channels, either for every frequency bin (square plots) or averaged across the frequency bins that MVPA could decode (scalp maps). For agreeableness, relations were strikingly consistent across the four conditions. Specifically, activity in the upper alpha band at posterior electrodes correlated negatively with agreeableness scores, whereas activity in the beta band was positively correlated with agreeableness scores. This was distributed mainly over parietal areas in eyes open conditions, and across both parietal and frontal electrodes in eyes closed conditions. We did not observe any obvious pattern of correlations for neuroticism.

A

Agreeableness and EEG power correlations
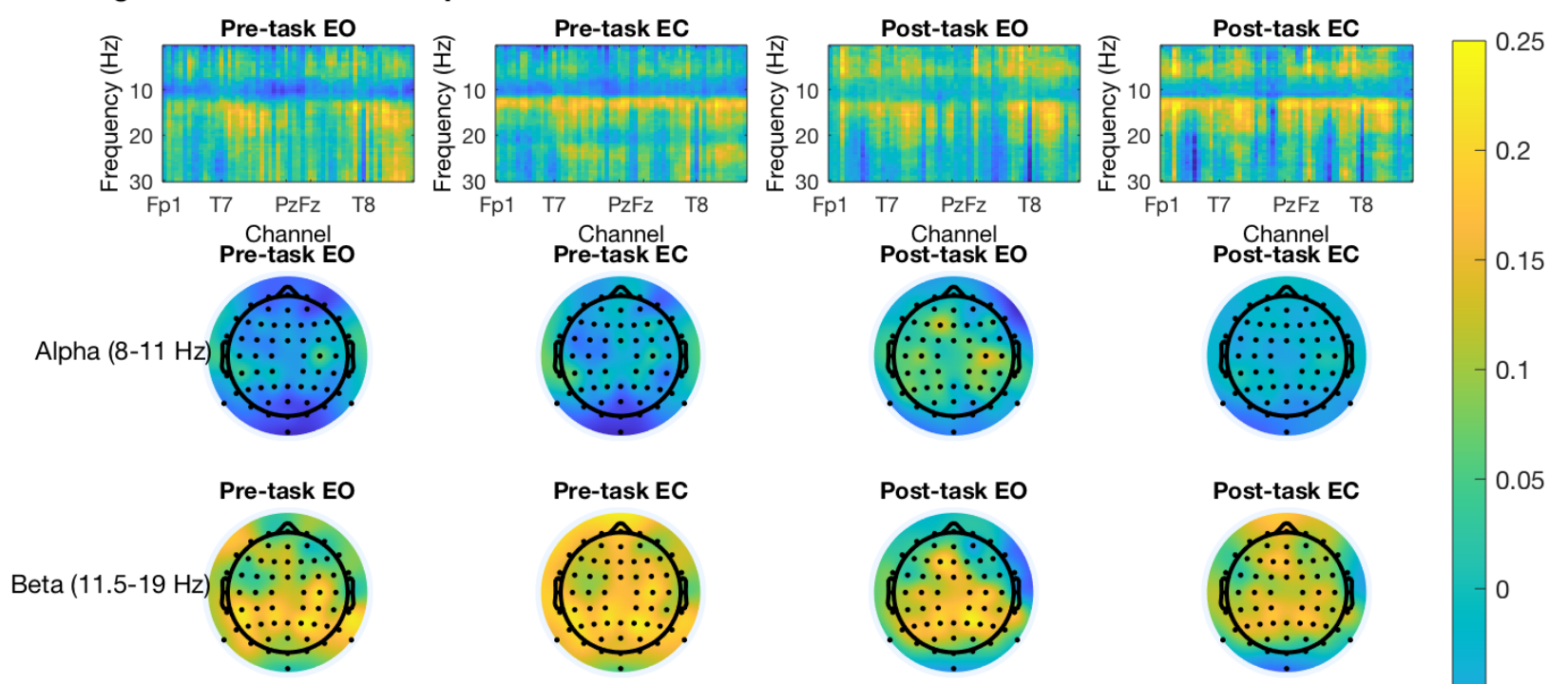

B
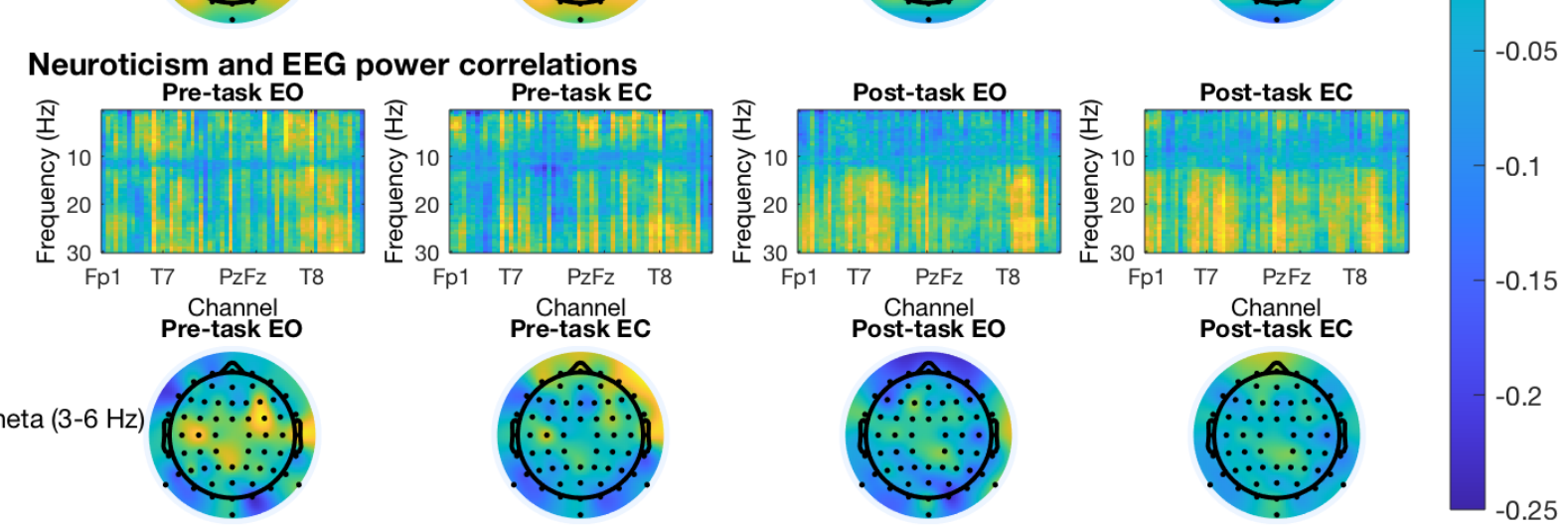

Correlation strength

Figure 3. Correlations between spectral power at each electrode and frequency bin and personality trait scores for A) agreeableness and B) neuroticism. Scalp maps display Spearman correlation coefficients averaged across selected frequency bands. EO = Eyes open, EC $=$ Eyes Closed. 


\section{Discussion}

In the current study we used MVPA to examine whether scores on any of the Big 5 personality traits could be predicted from spectral power of resting-state EEG. We found that agreeableness scores could be predicted based on EEG power in the alpha and lower beta bands (between $8-19 \mathrm{~Hz}$ ), and that neuroticism scores could be predicted using EEG power in the lower theta band (between 3-6 $\mathrm{Hz}$ ). Further analyses revealed that alpha power at posterior channels was negatively correlated with agreeableness, and parietal beta power was positively correlated with scores for this trait. These findings contribute new insights into the neural correlates of personality, particularly for agreeableness which, to our knowledge, has never before been linked to power in the beta band. If robust, the associations reported here will help to inform and constrain theory and research in personality neuroscience.

\subsection{Neural Correlates of Personality Trait Measures}

The primary motivation for conducting an exploratory study was the fledgling state of the literature concerning personality traits and resting state EEG. There is minimal direct prior research concerning the relation between oscillatory patterns and either agreeableness or neuroticism, either at rest or during a task, and the few studies that exist are considerably underpowered for analyzing individual differences (e.g., $N<60$, see Mar, Spreng, \& DeYoung, 2013 for discussion of this problem). It therefore did not make sense to formulate hypotheses about which traits would relate to which frequencies or brain regions. Rather, the lack of prior research made it appropriate to utilize data-driven methods that are inclusive of a wide range of oscillatory frequencies and brain regions. However, the lack of direct prior research to cross-reference also places obvious constraints on generating decisive explanations for the present observations. Until future research confirms our results with straightforward MVPA replications or targeted analyses relating a trait to a neural marker, we hold any inferences delicately. This does not detract from the value of our study for deriving tentative hypotheses to be tested in future work; indeed, observations such as we have made here are foundational and necessary before these hypotheses could be developed. With this caveat in mind, we now turn to potential explanations for our results, drawing on personality theory and prior research relating oscillatory patterns to relevant frequencies.

4.1.1 How and why are personality traits linked to neural data? Personality neuroscience recognizes that all individual differences in behavior, cognition, and emotion must be represented by corresponding differences in brain activity (Allen \& DeYoung, 2017).

\footnotetext{
${ }^{4}$ Finally, there is an additional artefactual explanation: it is possible that MVPA is detecting some increased motor movement not entirely removed by pre-processing (e.g., eye-blinks) that could meaningfully relate to the trait: for example, individuals higher in neuroticism looking around the room more due to nervousness. However, if eye blinks did
}

However, this provides little insight into how or why traits might be represented by neural data. Thoroughly parsing our results (and indeed, any data concerning neural correlates of personality) firstly requires consideration of what kinds of processes might, in principle, drive decoding performance.

Any relation between a neural measure and a personality trait can likely be explained in one of two ways. First, there could be behaviors, cognitions, or emotions occurring at the time that data is collected-that is, state processes-that individuals scoring higher on a personality trait are more likely to display. This possibility (that our neural measure is capturing one or more state processes) is in line with evidence that personality traits correspond well to density distributions of psychological states (Fleeson, 2001; Fleeson \& Gallagher, 2009). This explanation would suggest that the neural measure reflects aspects of momentary cognition or emotion to which individuals higher on the personality trait are susceptible.

Second, there could be some more enduring or stable differences in neural activity between those higher or lower on the trait that is captured by the neural measure. This possibility is more of an essentialist perspective aligning with classic theories concerning the biological bases of personality (e.g., Depue, 1995; Eysenck, 1967). This explanation suggests that the neural measure reflects a modulatory process that is present to a greater or lesser degree in those higher in the personality trait, that might influence momentary emotion, behavior, or cognition, but is not itself a reflection of these processes.

Of course, these two kinds of explanations are not mutually exclusive: enduring differences in neural activity may influence phasic responding, and neural plasticity allows for state processes to influence brain structure, connectivity, and general functioning (see Harmelech \& Malach, 2013, for an outline of this process as a possible explanation for variance in the resting state). 4 Nevertheless, the distinction between momentary state and more sustained or chronic processes is important if the ultimate aim is to derive a mechanistic understanding of the biological basis of personality (Allen \& DeYoung, 2017). We gain different insights from learning that we are measuring a momentary process versus a sustained modulatory factor that drives action tendencies. For example, chronic processes should be measurable during any situation, whereas state processes should only manifest in contexts that generate the relevant behavior, emotion, or cognition. Any proposed model of the links between the neural measure and behavior would thus be different when representing either of these explanations.

Both explanations are potentially congruent with the results obtained in the current study, and at present it is difficult to identify evidence that distinguishes between the two perspectives. As we measured participants at rest rather than performing a task, it might seem more likely that we detected a sustained process related to

contribute in the current study, we would be more likely to see correlations solely at frontal channels and decoding performance across a broad set of frequency bins, which was not the case. Future studies may wish to guard against this possibility by tracking eye movement and closely monitoring movement in the testing environment. 
agreeableness and neuroticism. However, no testing situation is truly neutral, and there may be elements of the resting state that trigger specific emotions, behaviors, and cognitions. For example, as trait agreeableness describes differences in prosocial tendencies (Graziano, Habashi, Sheese, \& Tobin, 2007; Habashi, Graziano, \& Hoover, 2016), it is possible that during data collection more agreeable individuals experience positive thoughts and emotions about donating their time towards advancing scientific knowledge. Conversely, disagreeable individuals may experience apathy or even resentment at the time spent sitting in a dark room with nothing to do. Likewise for neuroticism, which describes differences in negative emotionality, anxiety, and volatility (Widiger, 2009), it is possible that more neurotic individuals experience anxious thoughts and feelings whilst in an unfamiliar testing environment, and these more negative states are reflected in the patterns of neural activity being assessed. However, it is crucial to note that when assessing potential support for these possibilities we confront a many-to-one problem in both the personality and the EEG literatures. That is to say, there are many personality trait-relevant states that could be experienced by participants at any point in time (e.g., high-neuroticism participants in the resting state might feel low mood and disengagement instead of anxiety; highly agreeable participants might tend to feel polite and compliant instead of prosocial) and there is simultaneously a vast literature concerning theories of frequency band processes (for reviews see Başar, Başar-Eroğlu, Karakaş, \& Schürmann, 1999; Knyazev, 2007; Ward, 2003), many of which are relevant to personality traits and states.

If we were to assess the correspondence between prior EEG research and personality traits, we would invariably encounter this many-to-one problem. For example, Ertl, Hildebrandt, Ourina, Leicht, and Mulert (2013) found that frontal theta power was correlated with successful emotional regulation when watching aversive pictures. As individuals higher in neuroticism consistently demonstrate poorer emotion regulation strategies (John \& Gross, 2007; Kokkonen \& Pulkkinen, 2001; Yoon, Maltby, \& Joormann, 2013), this could support the possibility that we are decoding the relative ease or difficulty in regulating one's anxiety during the resting state scan. However, theta has been associated with a vast range of processes including cognitive control (Cavanagh \& Frank, 2014), memory retrieval and decision-making (Jacobs, Hwang, Curran, \& Kahana, 2006), memory encoding (Kirov, Weiss, Siebner, Born, \& Marshall, 2009), conscious awareness (Klimesch et al., 2001), and conflict between approach and avoidance (Shadli, Glue, McIntosh, \& McNaughton, 2015). Any of these phenomena could be framed, more or less directly, as being of relevance to neuroticism. Likewise, concerning agreeableness and the beta band, beta has been linked with processes such as motor function and motor imagery (Jenkinson \& Brown, 2011; Nakagawa et al., 2011; Feingold, Gibson, DePasquale, \& Graybiel, 2015), emotion (Güntekin \& Bașar, 2010; Senkowski, Kautz, Hauck, Zimmermann, \& Engel, 2011), and attention (Gao et al., 2017; Gola, Magnuski, Szumska, \& Wróbel, 2013), any of which could be have some relevance to agreeableness (for example, trying to remain still so as not to compromise the recording would be a process consistent with higher-agreeableness individuals, as would higher positive affect and higher attention). Thus, although we cannot rule out state responses simply because participants are providing data at rest, it would be premature to nominate the most likely momentary process that could be driving an effect and consequently develop targeted hypotheses from our results. The sections below address this issue by identifying several promising methods that could be used to gain greater clarity on these issues.

\subsection{Hypothesis Generation for Future Research}

4.2.1 Leveraging existing EEG individual differences research. EEG researchers increasingly acknowledge the impact of individual differences on EEG signals and processes. One particularly fruitful example of this is research concerning individual alpha peak frequency (Klimesch, 1997; 1999), which has been found to covary with measures of cognitive processing speed (Klimesch, 1999; but see Ouyang et al., 2020), fMRI measures of white matter architecture that may underlie patterns of functional connectivity (Valdés-Hernandez et al., 2010), and even perceptual processes, such as perceptual stability in binocular rivalry tasks (Katyal, He, He, \& Engel, 2019). The patterns of correlations between alpha and beta band power and agreeableness in our study might even be explained by shifts in alpha peak frequency. If higher alpha peak frequencies were present in individuals with high agreeableness, then we would expect to see larger power in the beta band and less power in the lower alpha band in those with high agreeableness scores, as we observed here. Future work could investigate the covariation between alpha power, alpha peak frequency, and personality traits. This avenue may be especially promising to investigate given that alpha power measures are thought to capture a fundamental aspect of how neural activity is coordinated across the brain (Klimesch, 2012; Mierau, Klimesch, \& Lefebvre, 2017). Such analyses should ideally be performed after accounting for individual differences in the $1 /$ f power spectrum, which can exert a large influence on alpha power measures (Ouyang et al., 2020).

4.2.2 Experimental approaches. An experimental approach may additionally help to identify the possible states that could drive above-chance decoding performance. First, researchers could assess emotions and other psychological states at intervals between restingstate data collection, and then use MVPA to predict those states in addition to personality traits. If the particular state (e.g., negative affect) is the reason why we are observing decoding performance for a given personality trait (e.g., neuroticism correlates with negative affect) then we would expect to observe stronger decoding performance for the state rather than the trait. If we do observe states that appear to be candidate processes underlying decoding accuracy, then subsequent studies could include a task designed to elicit a particular traitrelevant process alongside the resting state. If EEG differences are evoked by processes present in the moment, then we would expect that they would be induced 
to an even greater degree in a task designed to elicit them (e.g., for neuroticism, a fear induction paradigm).

\subsubsection{Decoding lower-order personality facets} and nuances. The current study assessed decoding performance for global personality traits. However, each trait can be decomposed into lower-order facets: for example, in the scale used for the current study, agreeableness can be divided into politeness and compassion; and neuroticism can be divided into volatility and withdrawal (DeYoung et al., 2007). Given that we did not have prespecified hypotheses for which traits could be decoded, we did not aim to assess the impact of individual facets. This also would have required us to conduct many more statistical tests, increasing the risk of spurious findings. However, future research can attempt to assess if specific facets within these traits are driving decoding performance. Given that facets within a trait are highly correlated with each other (as they must be, to form a general factor), these analyses should be conducted controlling for the other facet/s in the global trait. This approach could help to disentangle the tricky question of finding out whether it is a state response or trait regulatory process that we are detecting with MVPA: if one observed that only one facet in the global trait was driving decoding performance, then we would have some evidence against the regulatory process possibility (as some sort of regulatory process would be expected to affect shared variance across the facets). This could be taken even further by analyzing individual items in a scale (aka nuances; Mõttus, Kandler, Bleidorn, Riemann, \& McCrae, 2017), although this would come at the cost of increased measurement error.

4.2.4 Computational approaches. The final method is to build mathematical models that test the correspondence between the model's predictions and future data. There are many different ways that formal models can incorporate neural measures and processes (see Turner, Forstmann, Love, Palmeri, \& Van Maanen, 2017, for a review). Formal models are advantageous because researchers must specify all components of the model, including which neural level they wish to model (e.g., modelling state or phasic responses versus tonic or baseline responses-or the interconnections between these). Although these types of models are not frequently used in personality psychology, they have been effectively employed to investigate a theory linking extraversion with dopamine function (Wacker \& Smillie, 2015), by simulating the firing of dopamine neurons in the basal ganglia in response to reward signals (Pickering \& Pesola, 2014). For the current study, models could be built following results derived from investigations of individual alpha frequency or targeted state theories. Highly constrained hypotheses of how agreeableness or neuroticism relates to the brain regions, frequencies, or emotional states would be a valuable way to build from the initial empirical findings of the current research and future research.

\subsection{Future Uses of the Open Dataset.}

Our data and analysis scripts are available online, providing the opportunity for them to be used to address new research questions, or to top-up with additional data and investigate whether results are consistent with a larger sample. Note that although we did not perform inferential analyses on the data, we did thoroughly explore the data, making null hypothesis significance testing with our data plus a top-up sample inappropriate (Banks et al., 2019) unless the research question was very different from our own. However, given that there is current uncertainty over how many data points are required for machine learning decoding performance to stabilize, it could be highly valuable to re-run MVPA results with progressively different numbers of participants and observe consistency of estimates. It is possible that with larger samples, consistent effects will be observed for additional personality traits.

Additionally, because we computed MVPA analyses separately for each frequency bin, it was not possible to assess the dynamic coupling between different frequencies. This has been suggested as a source of individual differences in personality traits (Knyazev et al., 2003; Knyazev \& Slobodskaya, 2003; Knyazev, Schutter, \& van Honk, 2006). For example, Miskovic and colleagues (2010) found that slow- and fast-wave coupling in the resting state related to social anxiety (which relates to neuroticism; Kashdan, 2002). Future researchers could use similar methods to explore the present data, perhaps specifically focusing on neuroticism.

Finally, our analysis scripts can assist with replications of the current findings. If researchers have collected EEG resting data and personality measures across large samples, then it would be straightforward to adapt the analysis scripts that we have provided to assess whether finding related to agreeableness and neuroticism are consistent in an entirely new sample.

\section{Conclusion}

The current study is one of the first to use multivariate pattern analysis to decode personality traits from EEG power spectra. We found that agreeableness and neuroticism could be decoded across multiple testing conditions, with post-hoc analyses demonstrating consistent associations between agreeableness and the alpha/beta band. These initial results are foundational before future confirmatory investigations could be proposed, several of which we discussed in Section 4.2 above. We hope that this study provides clues for future research to understand how neurobiological factors drive individual differences in emotion, cognition, and behavior - that is, personality. 


\section{References}

Allen, T. A., \& DeYoung, C. G. (2017). Personality neuroscience and the five factor model. In T. A. Widiger (Ed.), The Oxford handbook of the five factor model (pp. 319-352). New York, NY: Oxford University Press. doi:10.1093/oxfordhb/9780199352487.013.26

Allport, G. W. (1937). Personality: A psychological interpretation. New York: Henry Holt \& Co.

Arlot, S., \& Celisse, A. (2010). A Survey of cross-validation procedures for model selection. Statistics Surveys, 4, 40-79. doi:10.1214/09-SS054

Banks, G. C., Field, J. G., Oswald, F. L., O’Boyle, E. H., Landis, R. S., Rupp, D. E., \& Rogelberg, S. G. (2019). Answers to 18 questions about open science practices. Journal of Business and Psychology, 34(3), 257-270. doi:10.1007/s10869-018-9547-8

Başar, E., Başar-Eroğlu, C., Karakaş, S., \& Schürmann, M. (1999). Are cognitive processes manifested in eventrelated gamma, alpha, theta and delta oscillations in the EEG? Neuroscience Letters, 259(3), 165-168. doi:10.1016/S0304-3940(98)00934-3

Başar, E., \& Güntekin, B. (2013). Review of delta, theta, alpha, beta, and gamma response oscillations in neuropsychiatric disorders. Supplements to Clinical Neurophysiology, 62, 303-341. doi:10.1016/B978-07020-5307-8.00019-3

Beaty, R. E., Chen, Q., Christensen, A. P., Qiu, J., Silvia, P. J., \& Schacter, D. L. (2018). Brain networks of the imaginative mind: Dynamic functional connectivity of default and cognitive control networks relates to openness to experience. Human Brain Mapping, 39(2), 811-821. doi:10.1002/hbm.23884

Beaty, R. E., Kaufman, S. B., Benedek, M., Jung, R. E., Kenett, Y. N., Jauk, E., ... \& Silvia, P. J. (2016). Personality and complex brain networks: the role of openness to experience in default network efficiency. Human Brain Mapping, 37(2), 773-779. doi:10.1002/hbm.23065

Bleidorn, W., Hopwood, C. J., \& Lucas, R. E. (2018). Life events and personality trait change. Journal of Personality, 86(1), 83-96. doi:10.1111/jopy.12286

Bleidorn, W., Kandler, C., \& Caspi, A. (2014). The behavioural genetics of personality development in adulthoodClassic, contemporary, and future trends. European Journal of Personality, 28(3), 244-255. doi:10.1002/per.1957

Bode, S., Feuerriegel, D., Bennett, D., \& Alday, P. M. (2019). The Decision Decoding ToolBOX (DDTBOX)-A multivariate pattern analysis toolbox for event-related potentials. Neuroinformatics, 17(1), 27-42. doi:10.1007/s12021-018-9375-z

Bode, S., Sewell, D. K., Lilburn, S., Forte, J. D., Smith, P. L., \& Stahl, J. (2012). Predicting perceptual decision biases from early brain activity. Journal of Neuroscience, 32(36), 12488-12498. doi:10.1523/JNEUROSCI.170812.2012

Brain Products GmbH (2017). BrainVision Analyzer User Manual. brainproducts.com/downloads.php?kid=9.

Buzsáki, G. (2006). Rhythms of the brain. New York, NY: Oxford University Press. doi:10.1093/acprof:oso/9780195301069.001.0001

Cauchoix, M., Barragan-Jason, G., Serre, T., \& Barbeau, E. J. (2014). The neural dynamics of face detection in the wild revealed by MVPA. Journal of Neuroscience, 34, 846-854. doi:10.1523/JNEUROSCI.3030-13.2014

Cavanagh, J. F., \& Frank, M. J. (2014). Frontal theta as a mechanism for cognitive control. Trends in Cognitive Sciences, 18(8), doi:10.1016/j.tics.2014.04.012
Cecere, R., Rees, G., \& Romei, V. (2015). Individual differences in alpha frequency drive crossmodal illusory perception. Current Biology, 25(2), 231-235. doi:10.1016/j.cub.2014.11.034

Chang, C., \& Lin, C-J. (2011). LIBSVM : A library for support vector machines. ACM Transactions on Intelligent Systems and Technology, 2(27), 1-27. doi:10.1145/1961189.1961199

Combrisson, E., \& Jerbi, K. (2015). Exceeding chance level by chance: The caveat of theoretical chance levels in brain signal classification and statistical assessment of decoding accuracy. Journal of Neuroscience Methods, 250, 126-136. doi:10.1016/j.jneumeth.2015.01.010

Cornew, L., Roberts, T. P., Blaskey, L., \& Edgar, J. C. (2012). Resting-state oscillatory activity in autism spectrum disorders. Journal of Autism and Developmental Disorders, 42(9), 1884-1894. doi:10.1007/s10803-0111431-6

Cronbach, L. J. (1951). Coefficient alpha and the internal structure of tests. Psychometrika, 16(3), 297-334. doi:10.1007/BF02310555

Delorme, A., \& Makeig, S. (2004). EEGLAB: An open source toolbox for analysis of single- trial EEG dynamics including independent component analysis. Journal of Neuroscience Methods, 134(1), 9-21. doi:10.1016/j.jneumeth.2003.10.009.

de Winter, J. C., Gosling, S. D., \& Potter, J. (2016). Comparing the Pearson and Spearman correlation coefficients across distributions and sample sizes: A tutorial using simulations and empirical data. Psychological Methods, 21(3), 273.

Depue, R. A. (1995). Neurobiological factors in personality and depression. European Journal of Personality, 9(5), 413-439. doi:10.1002/per.2410090509

DeYoung, C. G. (2006). Higher-order factors of the Big 5 in a multi-informant sample. Journal of Personality and Social Psychology, 91(6), 1138-51. doi:10.1037/00223514.91.6.1138

DeYoung, C. G., \& Gray, J. R. (2009). Personality neuroscience: Explaining individual differences in affect, behavior, and cognition. In P. J. Corr \& G. Matthews (Eds.), The Cambridge Handbook of Personality Psychology (pp. 323-346). New York: Cambridge University Press. doi:10.1017/CB09780511596544.023

DeYoung, C. G., Quilty, L. C., \& Peterson, J. B. (2007). Between facets and domains: 10 aspects of the Big Five. Journal of Personality and Social Psychology, 93(5), 880. doi:10.1037/0022-3514.93.5.880

Digman, J. M. (1990). Personality structure: Emergence of the five-factor model. Annual Review of Psychology, 41(1), 417-440. doi:10.1146/annurev.ps.41.020190.002221

Ertl, M., Hildebrandt, M., Ourina, K., Leicht, G., \& Mulert, C. (2013). Emotion regulation by cognitive reappraisalthe role of frontal theta oscillations. NeuroImage, 81 , 412-421. doi:10.1016/j.neuroimage.2013.05.044

Eysenck, H. J. (1967). The biological basis of personality. Springfield, IL: Charles C Thomas.

Feingold, J., Gibson, D. J., DePasquale, B., \& Graybiel, A. M. (2015). Bursts of beta oscillation differentiate postperformance activity in the striatum and motor cortex of monkeys performing movement tasks. Proceedings of the National Academy of Sciences, 112(44),

13687-13692. doi:10.1073/pnas.1517629112

Fleeson, W. (2001). Toward a structure-and processintegrated view of personality: Traits as density distributions of states. Journal of Personality and Social Psychology, 80(6), 1011. doi:10.1037/00223514.80.6.1011 
Fleeson, W., \& Gallagher, P. (2009). The implications of Big Five standing for the distribution of trait manifestation in behavior: Fifteen experience-sampling studies and a meta-analysis. Journal of Personality and Social Psychology, 97(6), 1097. doi:10.1037/a0016786

Gao, Y., Wang, Q., Ding, Y., Wang, C., Li, H., Wu, X., ... \& Li, L. (2017). Selective attention enhances beta-band cortical oscillation to speech under "Cocktail-Party" listening conditions. Frontiers in Human Neuroscience, 11, 34. doi:10.3389/fnhum.2017.00034

Gignac, G. E., \& Szodorai, E. T. (2016). Effect size guidelines for individual differences researchers. Personality and Individual Differences, 102, 74-78. doi:10.1016/j.paid.2016.06.069

Grandy, T. H., Werkle-Bergner, M., Chicherio, C., Lövdén, M., Schmiedek, F., \& Lindenberger, U. (2013). Individual alpha peak frequency is related to latent factors of general cognitive abilities. Neuroimage, 79, 10-18. doi:10.1016/j.neuroimage.2013.04.059

Gola, M., Magnuski, M., Szumska, I., \& Wróbel, A. (2013). EEG beta band activity is related to attention and attentional deficits in the visual performance of elderly subjects. International Journal of Psychophysiology, 89(3), 334341. doi:10.1016/j.ijpsycho.2013.05.007

Graziano, W. G., Habashi, M. M., Sheese, B. E., Tobin, R. M. (2007). Agreeableness, empathy, and helping: A person $\mathrm{X}$ situation perspective. Journal of Personality and Social Psychology, 93, 583-599. doi:10.1037/00223514.93.4.583

Grootswagers, T., Wardle, S. G., \& Carlson, T. A. (2017). Decoding dynamic brain patterns from evoked responses: A tutorial on multivariate pattern analysis applied to time series neuroimaging data. Journal of Cognitive Neuroscience, 29(4), 677-697. doi:10.1162/jocn_a_01068

Güntekin, B., \& Başar, E. (2010). Event-related beta oscillations are affected by emotional eliciting stimuli. Neuroscience Letters, 483(3), 173-178. doi:10.1016/j.neulet.2010.08.002

Habashi, M. M., Graziano, W. G., \& Hoover, A. E. (2016). Searching for the prosocial personality: A Big Five approach to linking personality and prosocial behavior. Personality and Social Psychology Bulletin, 42(9), 11771192. doi:10.1177/0146167216652859

Harmelech, T., \& Malach, R. (2013). Neurocognitive biases and the patterns of spontaneous correlations in the human cortex. Trends in Cognitive Sciences, 17(12), 606615. doi:10.1016/j.tics.2013.09.014

Harmon-Jones, E. (2003). Clarifying the emotive functions of asymmetrical frontal cortical activity. Psychophysiology, 40(6), 838-848. doi:10.1111/1469-8986.00121

Hogendoorn, H., Verstraten, F. A., \& Cavanagh, P. (2015). Strikingly rapid neural basis of motion-induced position shifts revealed by high temporal-resolution EEG pattern classification. Vision Research, 113, 1-10. doi:10.1016/j.visres.2015.05.005

Hughes, D. M., Yates, M. J., Morton, E. E., \& Smillie, L. D. (2015). Asymmetric frontal cortical activity predicts effort expenditure for reward. Social Cognitive and Affective Neuroscience, 10(7), 1015-1019. doi:10.1093/scan/nsu149

Jacobs, J., Hwang, G., Curran, T., \& Kahana, M. J. (2006). EEG oscillations and recognition memory: theta correlates of memory retrieval and decision making. Neuroimage, $32(2)$,

978-987. doi:10.1016/j.neuroimage.2006.02.018

Jenkinson, N., \& Brown, P. (2011). New insights into the relationship between dopamine, beta oscillations and motor function. Trends in Neurosciences, 34(12), 611618. doi:10.1016/j.tins.2011.09.003
John, O. P., \& Gross, J. J. (2007). Individual differences in emotion regulation. In J. J. Gross (Ed.), Handbook of emotion regulation. New York, NY: The Guilford Press.

John, O. P., Naumann, L. P., \& Soto, C. J. (2008). Paradigm shift to the integrative big five trait taxonomy. In O. P. John, R. W. Robins, \& L. A. Pervin (Eds.), Handbook of personality: Theory and research (pp. 114-158). New York, NY: The Guilford Press.

Kane, M. J., Gross, G. M., Chun, C. A., Smeekens, B. A., Meier, M. E., Silvia, P. J., \& Kwapil, T. R. (2017). For whom the mind wanders, and when, varies across laboratory and dailylife settings. Psychological Science, 28(9), 1271-1289. doi:10.1177/0956797617706086

Katyal, S., He, S., He, B., \& Engel, S. A. (2019). Frequency of alpha oscillation predicts individual differences in perceptual stability during binocular rivalry. Human Brain Mapping, 40(8), 2422-2433. doi:10.1002/hbm.24533

Kashdan, T. B. (2002). Social anxiety dimensions, neuroticism, and the contours of positive psychological functioning. Cognitive Therapy and Research, 26(6), 789-810. doi:10.1023/A:1021293501345

Kennis, M., Rademaker, A. R., \& Geuze, E. (2013). Neural correlates of personality: an integrative review. Neuroscience \& Biobehavioral Reviews, 37(1), 73-95. doi:10.1016/j.neubiorev.2012.10.012

Kirov, R., Weiss, C., Siebner, H. R., Born, J., \& Marshall, L. (2009). Slow oscillation electrical brain stimulation during waking promotes EEG theta activity and memory encoding. Proceedings of the National Academy of Sciences, 106(36), 15460-15465. doi:10.1073/pnas.0904438106

Klimesch, W. (1997). EEG-alpha rhythms and memory processes. International Journal of Psychophysiology, 26(1-3), 319-340.

Klimesch, W. (1999). EEG alpha and theta oscillations reflect cognitive and memory performance: a review and analysis. Brain Research Reviews, 29(2-3), 169-195. doi:10.1016/S0165-0173(98)00056-3

Klimesch, W. (2012). Alpha-band oscillations, attention, and controlled access to stored information. Trends in Cognitive Sciences, 16(12), 606-617. doi:10.1016/j.tics.2012.10.007

Klimesch, W., Doppelmayr, M., Schimke, H., Pachinger, T. (1996). Alpha frequency, reaction time, and the speed of processing information. Journal of Clinical Neurophysiology, 13, 511-518. doi:10.1097/00004691199611000-00006

Klimesch, W., Doppelmayr, M., Yonelinas, A., Kroll, N. E., Lazzara, M., Roehm, D., \& Gruber, W. (2001). Theta synchronization during episodic retrieval: neural correlates of conscious awareness. Cognitive Brain Research, 12(1), 33-38. doi:10.1016/S09266410(01)00024-6

Knyazev, G. G. (2007). Motivation, emotion, and their inhibitory control mirrored in brain oscillations. Neuroscience \& Biobehavioral Reviews, 31(3), 377-395. doi:10.1016/j.neubiorev.2006.10.004

Knyazev, G. G., Schutter, D. J., \& van Honk, J. (2006). Anxious apprehension increases coupling of delta and beta oscillations. International Journal of Psychophysiology, 61(2), 283-287. doi:10.1016/j.ijpsycho.2005.12.003

Knyazev, G. G., \& Slobodskaya, H. R. (2003). Personality trait of behavioral inhibition is associated with oscillatory systems reciprocal relationships. International journal of Psychophysiology, 48(3), 247-261. doi: 10.1016/S0167-8760(03)00072-2

Knyazev, G. G., Slobodskaya, H. R., Safronova, M. V., Sorokin, O. V., Goodman, R., \& Wilson, G. D. (2003). Personality, psychopathology and brain oscillations. Personality and 
Individual Differences, 35(6), 1331-1349. doi:10.1016/S0191-8869(02)00353-7

Kokkonen, M., \& Pulkkinen, L. E. A. (2001). Extraversion and neuroticism as antecedents of emotion regulation and dysregulation in adulthood. European Journal of Personality, 15(6), 407-424. doi:10.1002/per.425

Korjus, K., Uusberg, A., Uusberg, H., Kuldkepp, N., Kreegipuu, K., Allik, J., ... \& Aru, J. (2015). Personality cannot be predicted from the power of resting state EEG. Frontiers in Human Neuroscience, 9, 63. doi:10.3389/fnhum.2015.00063

Kuper, N., Käckenmester, W., \& Wacker, J. (2019). Resting Frontal EEG Asymmetry and Personality Traits: A Metaanalysis. European Journal of Personality. doi:10.1002/per.2197

MacCallum, R. C., Zhang, S., Preacher, K. J., \& Rucker, D. D. (2002). On the practice of dichotomization of quantitative variables. Psychological Methods, 7(1), 19. doi:10.1037/1082-989X.7.1.19

Mar, R. A., Spreng, R. N., \& DeYoung, C. G. (2013). How to produce personality neuroscience research with high statistical power and low additional cost. Cognitive, Affective, \& Behavioral Neuroscience, 13(3), 674-685. doi:10.3758/s13415-013-0202-6

Mierau, A., Klimesch, W., \& Lefebvre, J. (2017). Statedependent alpha peak frequency shifts: Experimental evidence, potential mechanisms and functional implications. Neuroscience, 360, 146-154. doi:10.1016/j.neuroscience.2017.07.037

Miskovic, V., Ashbaugh, A. R., Santesso, D. L., McCabe, R. E., Antony, M. M., \& Schmidt, L. A. (2010). Frontal brain oscillations and social anxiety: A cross-frequency spectral analysis during baseline and speech anticipation. Biological Psychology, 83(2), 125-132. doi:10.1016/j.biopsycho.2009.11.010

Mõttus, R., Kandler, C., Bleidorn, W., Riemann, R., \& McCrae, R. R. (2017). Personality traits below facets: The consensual validity, longitudinal stability, heritability, and utility of personality nuances. Journal of Personality and Social Psychology, 112(3), 474.

Nakagawa, K., Aokage, Y., Fukuri, T., Kawahara, Y., Hashizume, A., Kurisu, K., \& Yuge, L. (2011). Neuromagnetic beta oscillation changes during motor imagery and motor execution of skilled movements. Neuroreport, 22(5), 217-222. doi:10.1097/WNR.0b013e328344b480

Ouyang, G., Hildebrandt, A., Schmitz, F., \& Herrmann, C. S. (2020). Decomposing alpha and $1 / \mathrm{f}$ brain activities reveals their differential associations with cognitive processing speed. NeuroImage, 116304. doi:10.1016/j.neuroimage.2019.116304

Ozer, D. J., \& Benet-Martinez, V. (2006). Personality and the prediction of consequential outcomes. Annual Review of Psychology, 57, 401-421.

doi: 10.1146/annurev.psych.57.102904.190127

Pickering, A. D., \& Pesola, F. (2014). Modeling dopaminergic and other processes involved in learning from reward prediction error: contributions from an individual differences perspective. Frontiers in Human Neuroscience, 8, 740. doi:10.3389/fnhum.2014.00740

R Core Team (2018). R: A language and environment for statistical computing. R Foundation for Statistical Computing, Vienna, Austria. URL https://www.Rproject.org/.

Revelle, W. (2018) psych: Procedures for Personality and Psychological Research, Northwestern University, Evanston, Illinois, USA, https://CRAN.Rproject.org $/$ package $=$ psych Version $=1.8 .12$.

Schönbrodt, F. D., \& Perugini, M. (2013). At what sample size do correlations stabilize? Journal of Research in
Personality,

47, https://doi.org/10.1016/j.jrp.2013.05.009

609-612.

Senkowski, D., Kautz, J., Hauck, M., Zimmermann, R., \& Engel, A. K. (2011). Emotional facial expressions modulate pain-induced beta and gamma oscillations in sensorimotor cortex. Journal of Neuroscience, 31(41), 14542-14550. doi:10.1523/JNEUROSCI.6002-10.2011

Shadli, S. M., Glue, P., McIntosh, J., \& McNaughton, N. (2015). An improved human anxiety process biomarker: characterization of frequency band, personality and pharmacology. Translational Psychiatry, 5(12), e699. doi:10.1038/tp.2015.188

Smillie, L. D., Jach, H. K., Hughes, D. M., Wacker, J., Cooper, A. J., \& Pickering, A. D. (2019). Extraversion and rewardprocessing: Consolidating evidence from an electroencephalographic index of reward-predictionerror. Biological Psychology, 146, 107735. doi:10.1016/j.biopsycho.2019.107735

Soto, C. J. (2019). How replicable are links between personality traits and consequential life outcomes? The Life Outcomes of Personality Replication Project. Psychological Science, 30(5), 711-727. doi:10.1177/0956797619831612

Specht, J., Egloff, B., \& Schmukle, S. C. (2011). Stability and change of personality across the life course: The impact of age and major life events on mean-level and rankorder stability of the Big Five. Journal of Personality and Social Psychology, 101(4), 862. doi:10.1037/a0024950

Szucs, D., \& Ioannidis, J. (2017). When null hypothesis significance testing is unsuitable for research: a reassessment. Frontiers in Human Neuroscience, 11, 390. doi:10.3389/fnhum.2017.00390

Tong, F., \& Pratte, M. S. (2012). Decoding patterns of human brain activity. Annual Review of Psychology, 63, 483509. doi:10.1146/annurev-psych-120710-100412

Turner, B. M., Forstmann, B. U., Love, B. C., Palmeri, T. J., \& Van Maanen, L. (2017). Approaches to analysis in modelbased cognitive neuroscience. Journal of Mathematical Psychology, 76, 65-79. doi:10.1016/j.jmp.2016.01.001

Turner, W. F., Johnston, P., de Boer, K., Morawetz, C., \& Bode, S. (2017). Multivariate pattern analysis of event-related potentials predicts the subjective relevance of everyday objects. Consciousness and Cognition, 55, 46-58. doi:10.1016/j.concog.2017.07.006

Valdés-Hernández, P. A., Ojeda-González, A., MartínezMontes, E., Lage-Castellanos, A., Virués-Alba, T., ValdésUrrutia, L., \& Valdes-Sosa, P. A. (2010). White matter architecture rather than cortical surface area correlates with the EEG alpha rhythm. Neuroimage, 49(3), 23282339. doi:10.1016/j.neuroimage.2009.10.030

Wacker, J., Chavanon, M.-L., and Stemmler, G. (2010). Resting EEG signatures of agentic extraversion: new results and meta-analytic integration. Journal of Research in Personality, 44, 167-179. doi:10.1016/j.jrp.2009.12.004

Wacker, J., Chavanon, M. L., Leue, A., \& Stemmler, G. (2010). Trait BIS predicts alpha asymmetry and P300 in a Go/No - Go task. European Journal of Personality, 24(2), 85-105. doi:10.1002/per.740

Wacker, J., \& Smillie, L. D. (2015). Trait extraversion and dopamine function. Social and Personality Psychology Compass, 9(6), 225-238. doi:10.1111/spc3.12175

Ward, L. M. (2003). Synchronous neural oscillations and cognitive processes. Trends in Cognitive Sciences, 7(12), 553-559. doi:10.1016/j.tics.2003.10.012

Wickham, H. (2016) ggplot2: Elegant Graphics for Data Analysis. Springer-Verlag

New York. doi:10.1007/978-3-319-24277-4

Widiger, T. A. (2009). Neuroticism. In M. R. Leary \& R. H. Hoyle (Eds.), Handbook of individual differences in social 
behavior (pp. 129-146). New York, NY, US: The Guilford Press.

Yoon, K. L., Maltby, J., \& Joormann, J. (2013). A pathway from neuroticism to depression: examining the role of emotion regulation. Anxiety, Stress \& Coping, 26(5), 558572. doi:10.1080/10615806.2012.734810

Zhiyan, T., \& Singer, J. L. (1997). Daydreaming Styles, Emotionality and the Big Five Personality Dimensions. Imagination, Cognition and Personality, 16(4), 399-414. doi:10.2190/ATEH-96EV-EXYX-2ADB 